\title{
Evaluation of E-commerce Performance in SMEs based on Vector Auto Regression Model
}

\author{
Li Zhou ${ }^{1}$ and Qing-yi Chen ${ }^{2 *}$ \\ ${ }^{1}$ School of Management, MinzuUniversity of China,Beijing, China \\ ${ }^{2}$ School of Business, RenminUniversity of China,Beijing, China \\ ${ }^{1}$ zhoulimuc@163.com, ${ }^{2}$ qingyiruc@163.com
}

\begin{abstract}
With the development of information technology, E-business becomes the core competition of small and medium enterprises in recent years. In this paper, we make an empirical test to analyze how the application of E-business will effect on the business performance. The result shows that the E-business application index increased 1 percentage can drive sales volume increased by 0.22 percentages, so the effect of E-business to SMEs' business performance is obvious. From Johnson co-integration test, the result shows that there exist at least one direct co-integration relationship between E-business application and sales volume, which means that there exist a long-term equilibrium relationship between E-business application and sales volume in SMEs.
\end{abstract}

Keywords: E-commerce, business performance, small and medium enterprises (SMEs), vector auto regression model

\section{Introduction}

With the development of information technology, E-business becomes the core competition of Small and medium enterprises in recent years. E-business provides a new sales channel that can not consider business time and location, also online shopping will reduce the cost compared with traditional business[1]. Electronic business is an internet business, may be defined as the application of information and communication technologies (ICT) in support of all the activities of business. E-commerce focuses on the use of ICT to enable the external activities and relationships of the business with individuals, groups and other businesses[2]. E-business also promotes the development in small and medium enterprise (SMEs), as SMEs didn't have the scale competition advantage, E-business will become the core competition that can help SMEs get several advantages as lowest cost, differentiated services, and more convenient and efficient service[3]. According to the China statistical data the E-commerce market overall trade scale is 8.1 trillion RMB (\$1.3 trillion) in 2012, up $27.9 \%$. E-commerce has a huge potential in the retail market, also more small and medium enterprises begin to build their own online sales website.

The legal definition of "SMEs" varies by country and by industry, SMEs means a small business that having fewer than 500 employees for manufacturing businesses and less than $\$ 7$ million in annual receipts for most non manufacturing businesses. Hans Jansson (2012) pointed that the small business has several advantages as low cost and can be started on a part-time basis, small business is also well suited to internet marketing because it can easily serve specialized niches, independence is another advantage of owning a small business, as Ebusiness can further strengthen the advantages of small business, so that it would be important for SMEs to adopt E-business strategy[4]. 
E-business software solutions allow the integration of intra and inter firm business processes; it involves business processes spanning the entire value chain: electronic purchasing and supply chain management, processing orders electronically, handling customer service, and cooperating with business partners. Murray E.Jennex (2004) research the key infrastructure factors affecting the success of small companies in developing economies, the result shows that workers' skills, client interface, and technical infrastructure are the most important factors to the success of a B2B E-commerce relationship[5]. Christina A. Fader (2002) provided a model that identifies a number of unique factors that should be considered when estimating the optimal level of investment into an e-commerce initiative, and pointed out there are several constraints for SMEs to adopt E-commerce such as lack expertise in digital marketing and sales, insufficient resources required for ideal levels of investment and other creative cost and risk[6]. ElieElia and Louis-A (2007) presented empirical data from an electronic survey conducted among 96 manufacturing SMEs to investigate e-commerce initiatives and their related benefits. The research findings point to four main profiles of manufacturing SMEs with different e-commerce focuses[7].

As the E-business is becoming more and more important to small and medium sized enterprises (SMEs), many researchers focused on the marketing strategy of E-commerce and give out some suggestions in using e-business technology. PatriziaFariselli (1999) explored three issues as globalisation; SMEs and e-commerce, then pointed that there are important synergies between e-commerce (virtual) networks and (real) production networks[8]. A. J. Davies, A. J. Garcia-Sierra (1999) pointed out that electronic commerce has been accepted as a bona fide business practice in the commercial world, and monitored and researched the usage patterns and find out the effect of these technologies not only on the smaller companies independently, but also on their customers, suppliers and collaborators[9]. Erik Wierstra, Gabriele Kulenkampff (2001) assessed the impacts of basic elements of business strategies on the relative competitive position of selected types of ISPs, and find out that the incumbent telcos have a relatively strong starting point in the ISP market, while small regional ISPs have a weak starting point[10].

In recent years, e-commerce technology used more widely in SMEs. David A. Johnston, Lorna Wright (2004) pointed out that over 60\% of Small and Medium Enterprises (SMEs) in the USA and Canada have adopted some form of business process through a computer mediated network, such as the Internet[11]. Also, there are many researchers use enterprise models and empirical study to measure the impact of e-business to small business in emerging economics[12-14]. As the E-commerce developed fast in China, using E-commerce becomes more and more important to small and medium enterprise (SMEs). The small business has several advantages as low cost and can be started on a part-time basis, and E-business technology can strengthen these advantages. In this paper, we try to analyze how E-commerce application will affect the business performance in emerging countries as China and then give out some suggestions.

\section{Model Analysis}

\subsection{VAR Model}

Vector auto regression (VAR) is a statistical model used to capture the linear interdependencies among multiple time series. An estimated VAR model can be used for forecasting, and the quality of the forecasts can be judged, in ways that are completely analogous to the methods used in unvaried autoregressive modeling.VAR model is the simultaneous form of autoregressive model, A VAR (p) model of a time series y ( $t$ ) has the form as: 


$$
A_{0} y_{(t)}=A_{1} y_{(t-1)}+\cdots+A_{p} y_{(t-p)}+\varepsilon_{(t)}
$$

The structure of VAR model is decided only by the number of variables and the lag length. For example, if a VAR model has two variables as $\mathrm{y}_{1 \mathrm{t}}$ and $\mathrm{y}_{2 \mathrm{t}}$, as:

$$
\left\{\begin{array}{l}
y_{1, t}=c_{1}+\pi_{11.1} y_{1, t-1}+\pi_{12.1} y_{2, t-1}+\mu_{1 t} \\
y_{2, t}=c_{2}+\pi_{21.1} y_{1, t-1}+\pi_{22.1} y_{2, t-1}+\mu_{2 t}
\end{array}\right.
$$

Then we change this formula into matrix form, as:

$$
\left[\begin{array}{l}
y_{1 t} \\
y_{2 t}
\end{array}\right]=\left[\begin{array}{l}
c_{1} \\
c_{2}
\end{array}\right]+\left[\begin{array}{ll}
\pi_{11,1} & \pi_{12.1} \\
\pi_{21.1} & \pi_{22.1}
\end{array}\right]\left[\begin{array}{l}
y_{1, t-1} \\
y_{2, t-1}
\end{array}\right]+\left[\begin{array}{l}
\mu_{1 t} \\
\mu_{2 t}
\end{array}\right]
$$

Assume that

$$
Y_{t}=\left[\begin{array}{l}
\left.y_{1 t}\right\rceil \\
y_{2 t}
\end{array}\right], c=\left[\begin{array}{l}
c_{1} \\
c_{2}
\end{array}\right], \Pi_{1}=\left\lceil\begin{array}{ll}
\pi_{11.1} & \left.\pi_{12.1}\right\rceil \\
\pi_{21.1} & \pi_{22.1}
\end{array}\right], \mu_{t}=\left\lceil\begin{array}{l}
\left.\mu_{1 t}\right\rceil \\
\mu_{2 t}
\end{array}\right]
$$

So that, formula (3) can be written as:

$$
Y_{t}=c+\prod_{1} Y_{t-1}+\mu_{t}
$$

This is the basic model of VAR, as the formula only has lagged endogenous variables, so that these lagged endogenous variables are asymptotic unrelated with $\mu_{\mathrm{t}}$. Then we can use OLS method to estimate each VAR formula, and the parameter estimators that we gat will be consistent.

\subsection{Stability Conditions}

The stability of the VAR model means that when we put an impulse to the innovation of on formula in the VAR mode,the impact of the effect will gradually reduce .The basic condition of stability is that: all the eigenvalue of $\Pi_{1}$ should be located within the unit circle. According to the formula 5 , when $t=1$, it should be:

$$
Y_{1}=c+\prod_{1} Y_{0}+\mu_{1}
$$

And when $\mathrm{t}=2$, we calculate the formula with iterative method, as:

$$
Y_{2}=c+\Pi_{1} Y_{1}+\mu_{2}=\left(1+\Pi_{1}\right) c+\Pi_{1}^{2} Y_{0}+\Pi_{1} \mu_{1}+\mu_{2}
$$

So that, when $\mathrm{t}=\mathrm{t}$, it could be written as:

$$
Y_{t}=\left(1+\Pi_{1}+\Pi_{1}{ }^{2}+\cdots+\Pi_{1}{ }^{t-1}\right) c+\Pi_{1}{ }^{t} Y_{0}+\sum_{i=0}^{t-1} \Pi_{1}^{i} \mu_{t-i}
$$

From the formula above, we can get that $Y_{t}$ becomes a function to the vector $\mu, Y_{0}$ and $\mu_{t}$ after the formula transformation. So we can analysis the impact result of these vectors to find out whether the VAR model is stable. If the VAR model is stable, it will satisfy the conditions as: 
1) If give one unit impulse to $\mathrm{c}$ at $\mathrm{t}=1$, when $\mathrm{t} \rightarrow \infty$, the effect will have a Limit value as $\left(\mathrm{I}-\Pi_{1}\right)^{-1}$

2) If give one unit impulse to $Y_{0}$, the effect will be $\Pi_{1}{ }^{t}$ when $t=t$ and will be gradually disappeared with time has been increased.

From the analysis about VAR model, we can get that if the VAR model has the unit root, it will have the memory about impulse impact for a long time, so this VAR model is not stable. Also, the response ofendogenous variables will not reduce with time increased in this case.

\section{Empirical Analysis}

\subsection{Data Collection and Evaluation Index}

In order to analyze how the application of E-business effect on the business performance, we use STATA 12.0 software and make a statistical analysis of E-business application index (EB) and sales volume (SV) in SMEs from Beijing .

The E-business application index is very comprehensive, so that we use analytic hierarchy process (AHP) in order to construct this index. After the measurement and selection, we constructed the evaluation index of e-commerce development level in SMEs. This evaluation system mainly includes three class as e-commerce transactions Index, Informatization development Index and human capital Index. This E-business application index can be used to analyze the level of E-business application in SMEs, the content of these three indicators as shown below.

a) E-commerce transactions Index (X1): Share of e-commerce transactions, that is, the ratio of e-commerce transactions to the total turnover.

b) Informatization development Index(X2): mainly include the informatization investment ratio, the average daily efficiency of computer and the average hits ratio of enterprise website. The informatization investment ratio means the proportion of informatization investment to total investment.

c)Human capital Index(X3): staff ratio of the electronic commerce enterprise and the popularity of the Internet level.

The E-business application index can be calculated by using linear weighted method. According to the index system, we used comprehensive evaluation method to measure the Ebusiness application index (EAI), the calculation formula is:

$$
\begin{array}{r}
E A I=\sum_{i=1}^{m}\left(\sum_{j=1}^{n} P_{i j} W_{i j}\right) \times W_{i j}(9) \\
P_{i j}=\frac{x_{i j}}{\max x_{i j}} \times 100(10)
\end{array}
$$

In this formula, EAI represents the score of E-business application in SMEs, $\mathrm{P}$ is the calculated value of the indicators, and $\mathrm{W}$ is the corresponding weight.The weight of every indicator is calculated by AHP method, the weight is determined by the influence of each index to the upper level index, and we use 1-9 to rank the influence level.1means the influence level is same and 9 means the influence level is highest, the influence level is increased from 1 to 9. By calculating the weight, we get that the weights of three indicators as $\mathrm{W} 1=0.31$, $\mathrm{W} 2=0.49$ and $\mathrm{W} 3=0.20$. 
So that we can get the E-business application index based on this method. The data of sales volume is collected from Beijing statistic year book and Caixin database, period from 2001 to 2011. We also undertake log processing to data, noted as LnEB and LnSV.

\subsection{ADF Unit Root Test}

The unit root test was first put forward by David Dickey \& Wayne Fuller, so it is also called DF test. DF test is a basic method in stationarity test, if we have a model as:

$$
Y_{t}=\rho Y_{t-1}+\mu_{t}
$$

DF test is the significance test to the coefficient. If $\rho<1$, when $T \rightarrow \infty, \rho^{T} \rightarrow 0$, that means the impulse will be reduced when the time is increased. However, if $\rho \geq 1$, the impulse will not be reduced with the time, so that this time-series data is not stable. The basic DF test model can be written as:

$$
Y_{t}=\beta_{1}+\beta_{2} t+(1+\delta) Y_{t-1}+\mu_{t}
$$

If we add the lagged variable of $\Delta Y_{t}$ in formula 10 , then it will be called the augmented Dickey-Fuller test, so that ADF test model can be written as:

$$
\Delta Y_{t}=\beta_{1}+\beta_{2} t+\delta Y_{t-1}+\alpha_{i} \sum_{i=1}^{m} \Delta Y_{t-i}+\varepsilon_{t}
$$

Data stable is the premise of establishing VAR model, an augmented Dickey-Fuller test (ADF) is a test for a unit root in a time series sample. We use ADF unit root test to inspect LnEB and LnSV, the result as is shown in Table 1. Through the test results we can see that LnEB and LnSV are non-stationary,then we test on d.LnEB and d.LnSV and demonstrate that d.LnEB and d.LnSV are stable, so we can build the VAR model and use granger test and cointegration test.

Table 1. Augmented Dickey-Fuller Test (ADF)

\begin{tabular}{|c|c|c|c|c|c|}
\hline Variable & Test Statistic & $\begin{array}{c}1 \% \text { Critical } \\
\text { Value }\end{array}$ & $\begin{array}{c}5 \% \text { Critical } \\
\text { Value }\end{array}$ & $\begin{array}{c}10 \% \text { Critical } \\
\text { Value }\end{array}$ & Result \\
\hline LnEB & -1.788 & -0.433 & -2.983 & -2.623 & Unstable \\
\hline LnSV & 1.305 & -0.566 & -2.983 & -2.623 & Unstable \\
\hline D. LnEB & -4.821 & -3.215 & -2.983 & -2.623 & Stable \\
\hline D. LnSV & -3.065 & -3.400 & -2.983 & -2.623 & Stable \\
\hline
\end{tabular}

\subsection{VAR Model}

Vector auto regression (VAR) is a statistical model used to capture the linear interdependencies among multiple time series. An estimated VAR model can be used for forecasting, and the quality of the forecasts can be judged.VAR model is the simultaneous form of autoregressive model, A VAR (p) model of a time series y ( $t$ ) has the form:

$$
A_{0} y_{(t)}=A_{1} y_{(t-1)}+\cdots+A_{p} y_{(t-p)}+\varepsilon_{(t)}
$$


In this paper, I use AIC, SC criterion to identify the lag length. From the result, we can get that the minimum AIC is in lag 2, so I choose lag 2 as the lag length. Then, we bulid the VAR model of LnEB and LnSV as:

$$
\operatorname{LnS} V=2.59+0.22 \operatorname{LnEB}_{t-1}+0.128 \mathrm{LnEB}_{t-2}+0.451_{\mathrm{LnSV}} \mathrm{L}_{t-1}+0.329 \mathrm{LnSV}_{t-2}
$$

According to this formula, we can get that the application of E-business will promote sales volume increase. LnEB at lag 1 period increased one percentage can drive LnSV increased by 0.22 percentages, so the effect of E-business to SMEs' business performance is obvious.

In order to analyze the relations between E-business application and sales volume, we use granger causality test to analyze this VAR model, the result is shown in table 2.From Table 2, we can get that LnEB is the reason to LnSV, which means E-business application is the reason to sales volume increase. However, LnSV is not the reason for LnEB, so that sales volume increase is not the reason to E-business; this is also same to the conclusion above.

Table 2. Granger Causality Test

\begin{tabular}{|c|c|c|c|c|}
\hline Equation & Excluded & chi2 & df & Prob $>$ chi2 \\
\hline LnEB & LnSV & 2.5005 & 2 & 0.286 \\
\hline LnSV & LnEB & 17.214 & 2 & 0.000 \\
\hline
\end{tabular}

At the same time, we take Johnson co-integration test to analyze the long-term relations between E-business application and sales volume increase, the results is shown in table 3 Co integration is a statistical property of time series variables. Two or more time series are co integrated if they share a common stochastic drift, if two or more series are individually integrated but some linear combination of them has a lower order of integration, then the series are said to be co integrated.

Table 3. Johnson Co-integration Test

\begin{tabular}{|c|c|c|c|c|c|}
\hline Rank & Parms & LL & Characteristic Value & Statistic & 5\% Significant level \\
\hline 0 & 6 & 25.653892 & & $8.1213^{*}$ & 15.41 \\
\hline 1 & 9 & 29.678554 & 0.63438 & 0.0720 & 3.76 \\
\hline
\end{tabular}

According to the results, there exist at least one direct co-integration relationship between E-business application and sales volume, which means that there exist a long-term equilibrium relationship between E-business application and small business sales volume.

\subsection{Impulse-response Analysis}

According to the results above, we can get that there exist a long-term equilibrium relationship between E-business application and small business sales volume, and E-business is the reason to business sales volume increase, also the VAR model is stable. In order to analyze the VAR model, I use Impulse-response function and cholesky variance decomposition, the results is shown in Figure 1 and Figure 2.

From Figure 1, we can get that when LnEB received one unit impact, it will lead LnSV increase currently, LnSV will reach the max at $\mathrm{t}=4$ period and begin to be stable then. It illustrates there is long-term effect between E-business application and small business sales volume. According to the impulse analysis results, we can get that E-business application will 
significant influence business sales volume increase, so that it is important to apply E-business in SMEs. The cholesky variance decomposition also shows the same result, the contribution degree of LnSV to LnSV is gradually reduced and the contribution degree of LnEB to LnSV is gradually increased. From figure 2, we find the contribution degree of LnSV to LnSV at $\mathrm{t}=1$ period is almost $100 \%$, and then reduced gradually from setp 2 , finally reduced to $67.2 \%$ in $\mathrm{t}=8$ period. At the same time, the contribution degree of $\mathrm{LnEB}$ to $\mathrm{LnSV}$ is $25 \%$ at $\mathrm{t}=1$ period, then increased and become stable from step 2, the contribution degree in $\mathrm{t}=8$ period is $62 \%$. This means that E-business application has a important contribution degree to sales volume increase, and can be used to explain the business performance in SMEs by using E-business technology.
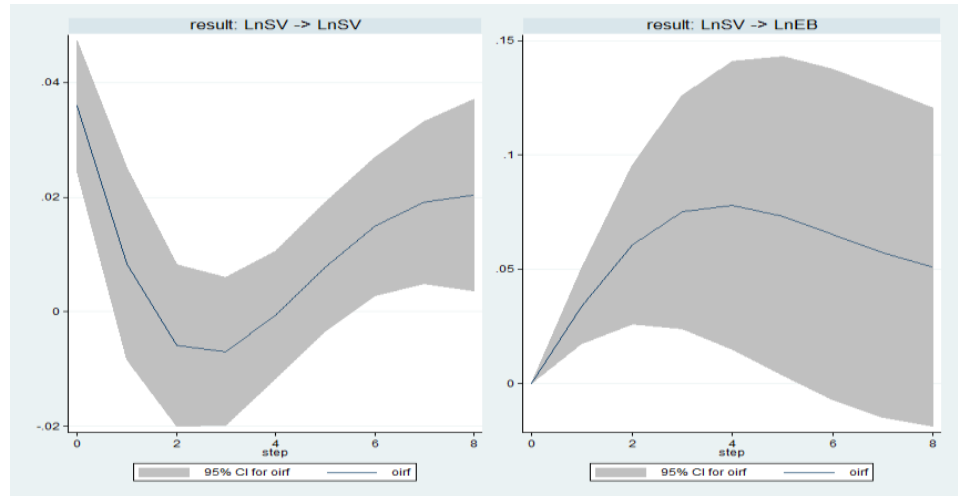

Figure 1. Impulse-response Analysis

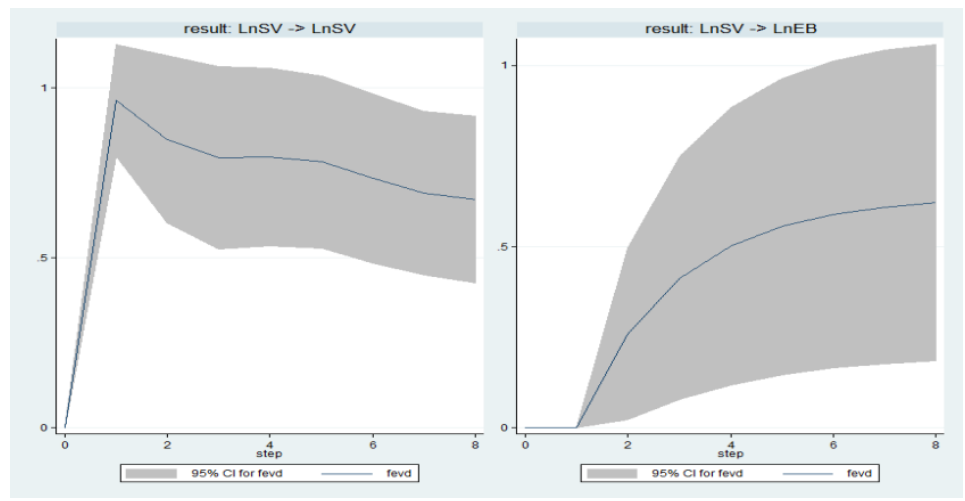

Figure 2.Cholesky Variance Decomposition

\section{Conclusion}

In this paper, we first introduce the ECRM and E-business strategy in enterprise, and use VAR model to analyze how E-commerce affect the business performance in emerging countries as China. The result shows that E-business application can improve the sales performance of small business obviously, there exist a long-term equilibrium relationship between E-business application and small business sales volume. E-business would be the most important factors for SMEs to success. The advantage of using E-business in SMEs basically has the following aspects: First, electronic commerce has been the market development tools, the network marketing activities of enterprises can improve marketing efficiency and reduce the cost of sales. For example, internet advertising can increase the sales 
about 10 times compared with the traditional advertising, but the cost is only $1 / 10$ of traditional advertising; Second, the electronic commerce can reduce procurement costs because the enterprise can seek the most preferential prices in the global market suppliers, and reduce the loss of intermediate links due to inaccurate information; Third, Electronic commerce as a marketing platform, the network transaction does not need an intermediary party, and can improve efficiency. By using the network information technology, customers' order can be received directly through the network, so that the products do not need to be stored to the warehouse but can be shipped directly to the customers. In addition, relying on the Internet technology like MSN, and other business real-time software dialog, it can strengthen the communication.

However, there are still some inhibitors of using e-commerce in SMEs, such as:

- The high cost of implementation

- Lack of organizational readiness with many SMEs having limited existing IT resources

- There are still some complex technologies like EDI which could require new skills

- Difficult to achieve timely network update

- The different perception between webpage description and the reality of goods

- Security, including confidentiality and fraud

Thus, a range of issues may affect SMEs decisions to invest in e-business and to take advantage of future opportunities. These inhibitors of using e-commerce in SMEs still need to analyze and research. With the change of market environment, enterprises have realized the importance of customer resource. E-CRM is a new management mechanism that aims to improve the relationship between enterprises and customers, it provides comprehensive, personalized customer information to sales and service personnel, it also strengthen the tracking service, information analysis capabilities, enabling them to build and maintain the" one-to-one relationship" between customers and enterprise. With the extensive application of management information system, small business will face more customer data, using a reasonable CRM strategy and information technology will help SMEs establish a long-term customer relationship and improve the business performance.

\section{References}

[1] H.Jinghua and J.Ximin, "An e-commerceperformance assessment model: Its development andan initial test on e-commerce applications in the retailsector of China", Information \& Management,vol.46,(2009),pp.100108.

[2] G. C.Juan, J.Daniel and M. Eusebio, "Implementinge-business through organizational learning:An empirical investigation in SMEs”,InternationalJournal of Information Management, vol.27,(2007), pp.173-186.

[3] F.Patrizia, O.Christinea and P. Christian, "Electronic commerce and the future for SMEsin a global marketplace: Networking and publicpolicies", Small Business Economics, vol.12,(1999), pp.261-275.

[4] M.Hilmersson,H.Jansson, "International network extension processes to institutionally different markets: Entry nodes and processes of exporting SMEs”,International Business Review,vol.21,(2012), pp.682-693.

[5] M.E. Jennex, D. Amoroso and O.Adelakun, "E-Commerce Infrastructure Success Factors for Small Companies in Developing Economies", Electronic Commerce Research,vol.4, (2004), pp.263-286.

[6] C.A. Fader,"Optimizing sunk investments in e-commerce: a quality assurance challenge for small businesses",International Journal on Digital Libraries,vol.3,(2002), pp.279-283.

[7] E.Elie and A. L.Louis, "Focus of B-to-Be-commerce initiatives and related benefits inmanufacturing smalland medium-sized enterprises",Information Systems and e-Business Management, vol.5,(2007), pp.1-23.

[8] F.Patrizia, O.Christine and P. Christian, "Electronic commerce and the future for SMEsin a global marketplace: Networking and publicpolicies”,Small Business Economics, vol.12,(1999),pp.261-275. 
[9] A.J. Davies and G.Sierra,"Implementingelectronic commerce in SMEs--Three casestudies", BT Technology Journal, vol.17,(1999),pp.97-111.

[10] W.Erik, K.Gabriele, "A frameworkforanalysing strategies of Internet ServiceProviders",NETNOMICS: Economic Researchand Electronic Networking,vol.3,(2001), pp.35-65.

[11] A. J.DavidandW. Lorna,"The e-businesscapability of small and medium sized firms in internationalsupply chains", Information Systems ande-Business Management, vol.2,(2004), pp.223-240.

[12] S.Phavaphan andK.Donyaprueth, "Effectsof e-CRM on customer-bank relationship qualityand outcomes: The case of Thailand", TheJournalof High Technology Management Research, vol.22,(2011),pp.141-157.

[13] F. Sylvie andJ.Judith, "Identifyingsuccess factors for rapid growth in SME e-commerce,"Small Business Economics, vol.19,(2002),pp.51-62.

[14] P. Lucia andS.Francesca, "Globalisation, EBusinessand SMEs: Evidence from the Italian districtof Prato", Small Business Economics, vol.22,(2004), pp.333-347.

[15] H.T.JamesandW.Richard, "A model ofe-commerce use by internationalizing SMEs",Journalof International Management, vol.7, (2001), pp.211-233.

[16] W.Ing-Long, H.Ching-Yi, "A strategy-based process for effectively determining systemrequirements in eCRM development”, Informationand Software Technology, vol.51,(2009), pp.1308-1318. 
International Journal of u-and e-Services, Science and Technology Vol.7, No.5 (2014) 